\title{
A Quantum Mechanics Treatment of Electron Diffraction by a Slit
}

\section{Francisco Lovey}

Centro Atómico Bariloche (CNEA). Bariloche, Argentina.

Corresponding author: franciscolovey@gmail.com

The diffraction of particles (electrons, neutrons, protons, atoms) by slits is a phenomenon that should be treated in the frame of quantum mechanics (QM) by solving the Schrodinger equation. However in most cases the Huygens principle and/or the Kirchhoff and Fresnel formalisms have been used.

An approach to apply QM to the diffraction of particles by a single or double slit was reported in reference [1]. However they proposal can only be applied when the opaque plate containing the slits has a negligible thickness. More recently in another report [2] the diffraction of neutrons by slits was analyzed. These authors considered the slit as an infinite potential well. The particles when traversing the slit must occupy the quantum states due to this well potential. Nevertheless, the authors fail giving a complete QM description as they applied the classic Kirchhoff formulation at the exit plane of the slit.

In this work we present a complete and simple QM formulation for the Fraunhofer diffraction of particles by a slit, that allow to identify the relation of the characteristics of the diffraction patterns with the eigenstates of a particle in an infinite potential well and also permit to take into account the effect of the thickness of the opaque plate containing the slit.

We consider an incident free particle wave function with wave vector $K$ along de $x$ axis, normal to the slit plane. The width (aperture) of the slit along the $y$ axis is $a$. The thickness of the opaque plate along the $x$ axis is $d$. The slit extends infinitely along de $z$ axis. We assume the wave length of the incoming particles to be rather much shorter than the slit width $a$.

The general solution inside the slit, that satisfy the continuity condition at the entrance plane $(x=0)$, may be formed by a linear combination of the even eigenstates of the particle in an infinite potential well. The odd solutions are excluded as they do not match with the boundary conditions. We get:

$\psi_{I I}(x, y)=\sum_{n} G_{n} e^{i \sqrt{\left(K^{2}-k_{y n}^{2}\right)} x} \sqrt{\frac{2}{a}} \cos k_{y n} y$

Where: $k_{y n}=(2 n+1) \pi / a, n=0,1,2, \ldots, \infty, k_{x n}^{2}+k_{y n}^{2}=K^{2}$ and $G_{n}=\frac{2 \sqrt{2}}{\pi} \frac{(-1)^{n}}{(2 n+1)}$

For $x>d$ we have solutions of free particles. We construct the general solutions in order to match with the function inside the slit evaluated at $x=d$. The total wave function reads:

$\psi_{I I I}(x, y)=\frac{1}{\sqrt{2 \pi}} \int_{-\infty}^{\infty} A\left(k_{y}\right) e^{i\left[\left(k_{x}(x-d)+k_{y} y\right]\right.} d k_{y} \quad k_{x}^{2}+k_{y}^{2}=K^{2}$

Where: $A\left(k_{y}\right)=\sum_{n} G_{n} e^{i \sqrt{\left(K^{2}-k_{y n}^{2}\right)} d} Q_{n}\left(k_{y}\right) \quad$ and $\quad Q_{n}\left(k_{y}\right)=\frac{1}{\sqrt{2 \pi}} \sqrt{\frac{2}{a}} \int_{-a / 2}^{a / 2} \cos k_{y n} y_{a} e^{-i k_{y} y_{a}} d y_{a}$ 
The function $Q_{n}\left(k_{y}\right)$ is the Fourier transform of the eigenstates in the slit. Therefore $\left|Q_{n}\left(k_{y}\right)\right|^{2}$ gives the density of probability to have a given direction of the component $p_{y}=\hbar k_{y}$ of the linear momentum for the $n^{\text {th }}$ state in the slit. We found $\left\langle k_{y}>=0\right.$ and $\left\langle k_{y}^{2}>=k_{y n}^{2}\right.$.

Finally the Fraunhofer diffraction intensity is given by the expression $\left|A\left(k_{y}\right)\right|^{2}$. We get explicitly:

$\left|A\left(k_{y}\right)\right|^{2}=\left|\frac{\sqrt{2 a}}{\pi^{3 / 2}} \sum_{n} \frac{(-1)^{n}}{(2 n+1)} e^{i \sqrt{\left(K^{2}-k_{y n}^{2}\right)}} \mathrm{d}\left\{\frac{\operatorname{sen}\left[\left(k_{y}-k_{y n}\right) a / 2\right]}{\left(k_{y}-k_{y n}\right) a / 2}+\frac{\operatorname{sen}\left[\left(k_{y}+k_{y n}\right) a / 2\right]}{\left(k_{y}+k_{y n}\right) a / 2}\right\}\right|^{2}$

We consider first the thickness of the opaque plate to be: $d<<a^{2} / \lambda$.

The expression (3) in this approximation is plotted in Fig. 1a.
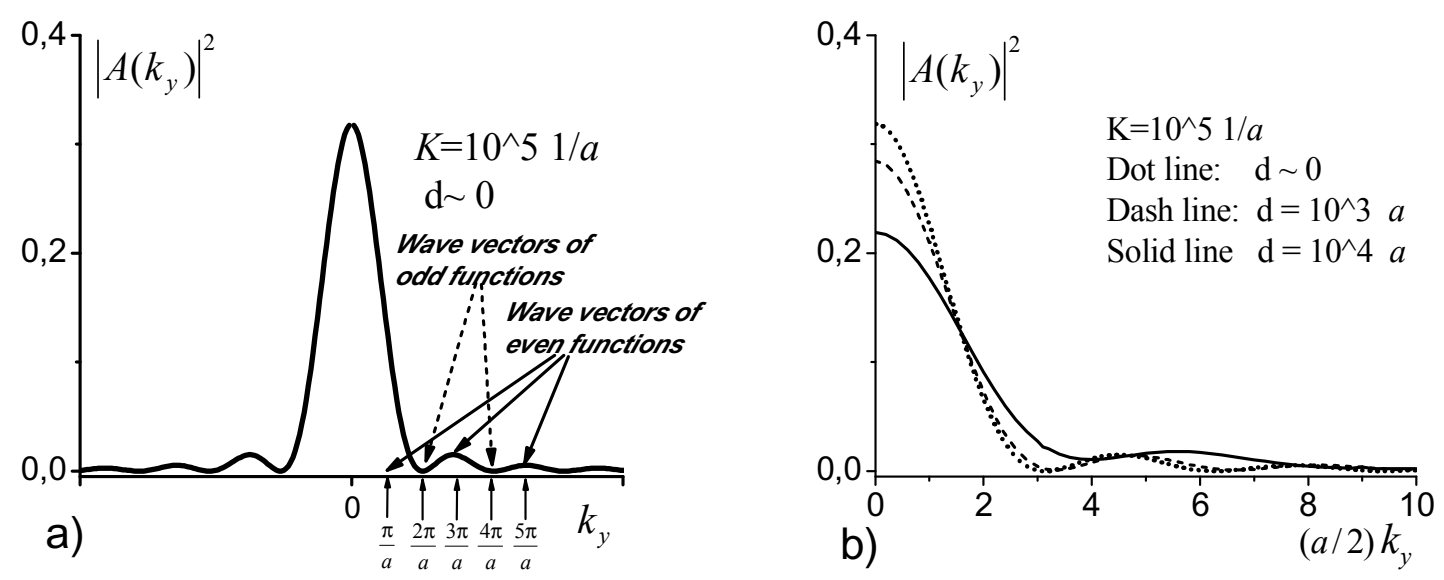

Figure 1: a) Fraunhofer diffraction for negligible $d$. b) The effect of the thickness $d$ on the diffraction.

The following characteristics can be remarked from Fig. 1a. $i$ ) The principal maximum corresponds to the mean value of the momentum $\left\langle k_{y}\right\rangle=0$ given by the set of functions $Q_{n}\left(k_{y}\right)$ in (2). ii) The secondary maxima appear for the wave vectors corresponding to the eigenstates in the equation (1). iii) The minima are associated to the odd solutions excluded by the boundary conditions.

The effect of the opaque plate thickness can be observed in Fig. 1b. The diffraction curve spread out as the thickness $d$ increases because the coefficients of the function in (3) are modified by the exponential factor. An estimated threshold to clearly observe the thickness effect is: $\lambda d / a^{2}>10^{-2}$.

\section{REFERENCES}

[1] T V Marcella, Eur. J. Phys. 23 (2002), p. 615.

[2] X Y Wu et al., Int. J. Modern Phys. B 23 (2009), p. 3255. 